\title{
Double Trouble - Spontaneous Coronary Artery Dissection of the Left Anterior Descending and Posterior Descending Arteries in a Right Dominant Circuit: A Case Report
}

\author{
Anton Mararenko a, c, Greg Minassian ${ }^{\mathrm{a}}$, Anshu Kataria ${ }^{\mathrm{a}}$, Firas Ajam ${ }^{\mathrm{b}}$, Matthew S. Schoenfeld ${ }^{\mathrm{b}}$
}

\begin{abstract}
Acute myocardial infarction is a condition that classically presents with chest pain and corresponding biomarkers and changes on electrocardiogram. Although most causes of acute coronary syndrome are due to acute plaque rupture resulting in coronary thrombosis, an increasingly prevalent condition known as spontaneous coronary artery dissection (SCAD) is becoming more commonly diagnosed. SCAD is characterized by a tear in the tunica media resulting in an intramural hematoma. Depending on the size of the hematoma, progressive extension can ultimately lead to coronary occlusion. Our team presents a 52-year-old female patient that presented with substernal chest pain and positive cardiac enzymes. Urgent coronary catheterization revealed bilateral SCAD involving the left anterior descending and posterior descending arteries in a right coronary dominant circuit. Our patient was treated with medical therapy alone and was safely discharged to home after close monitoring in the coronary care unit. Our team hopes to contribute to a growing body of evidence that bilateral SCAD can occur and can be successfully treated without percutaneous coronary intervention.
\end{abstract}

Keywords: Spontaneous coronary artery disease; Cardiology; Acute coronary syndrome; NSTEMI

\section{Introduction}

Spontaneous coronary artery dissection (SCAD) is becoming a more commonly identified cause of non-atherosclerotic acute coronary syndrome (ACS). Since it was first described in a

Manuscript submitted August 28, 2021, accepted October 5, 2021

Published online December 2, 2021

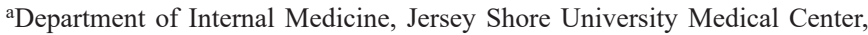
Neptune, NJ, USA

${ }^{b}$ Department of Cardiology, Jersey Shore University Medical Center, Neptune, NJ, USA

${ }^{\mathrm{c} C}$ Corresponding Author: Anton Mararenko, Department of Internal Medicine, Jersey Shore University Medical Center, Neptune, NJ, USA.

Email: Anton.Mararenko@hmhn.org

doi: https://doi.org/10.14740/jmc3774 young female patient found to have SCAD after retching, the medical community is becoming more aware of this potentially life-threatening condition [1]. The core pathophysiology of SCAD involves the formation of an intramural hematoma. Depending on the size and extent of dissection, the intramural hematoma can eventually form an occlusion resulting in myocardial ischemia [2]. SCAD presents similarly to ACS with a chief complaint of chest pain, positive cardiac biomarkers, and electrocardiogram changes including ST deviations [2]. The management of SCAD differs from traditional ACS secondary to acute plaque rupture as the coronary vessels are very friable due to damage of the tunica media. The left anterior descending (LAD) artery is most commonly affected; however, multivessel SCAD has been reported as well [3]. Most cases of SCAD are managed medically and have favorable outcomes [4].

\section{Case Report}

\section{Investigations}

Our team presents a 52-year-old female patient with a past medical history significant for hyperlipidemia, hypertension, and carotid stenosis who initially presented to an outside hospital with 1 day of non-radiating, substernal chest pain rated as $7 / 10$ in intensity with no alleviating or aggravating factors. The chest pain was associated with shortness of breath and palpitations. Prior to admission, the patient had a nuclear stress test that was negative for any signs of ischemia. Home medications included aspirin $81 \mathrm{mg}$ and atorvastatin $40 \mathrm{mg}$ daily.

\section{Diagnosis}

At the outside hospital, the patient was diagnosed with a nonST elevation myocardial infarction as the troponin had peaked at $0.45 \mathrm{ng} / \mathrm{mL}$ (reference $<0.04 \mathrm{ng} / \mathrm{mL}$ ). They were initially treated with beta blockade and nitroglycerine for angina as well as intravenous heparin drip for anticoagulation. Once the symptoms had stabilized, the patient was transferred to our institution for emergent left heart catheterization. Upon presentation, the patient was found to have mild chest pain that was largely improving. The patient was hemodynamically 

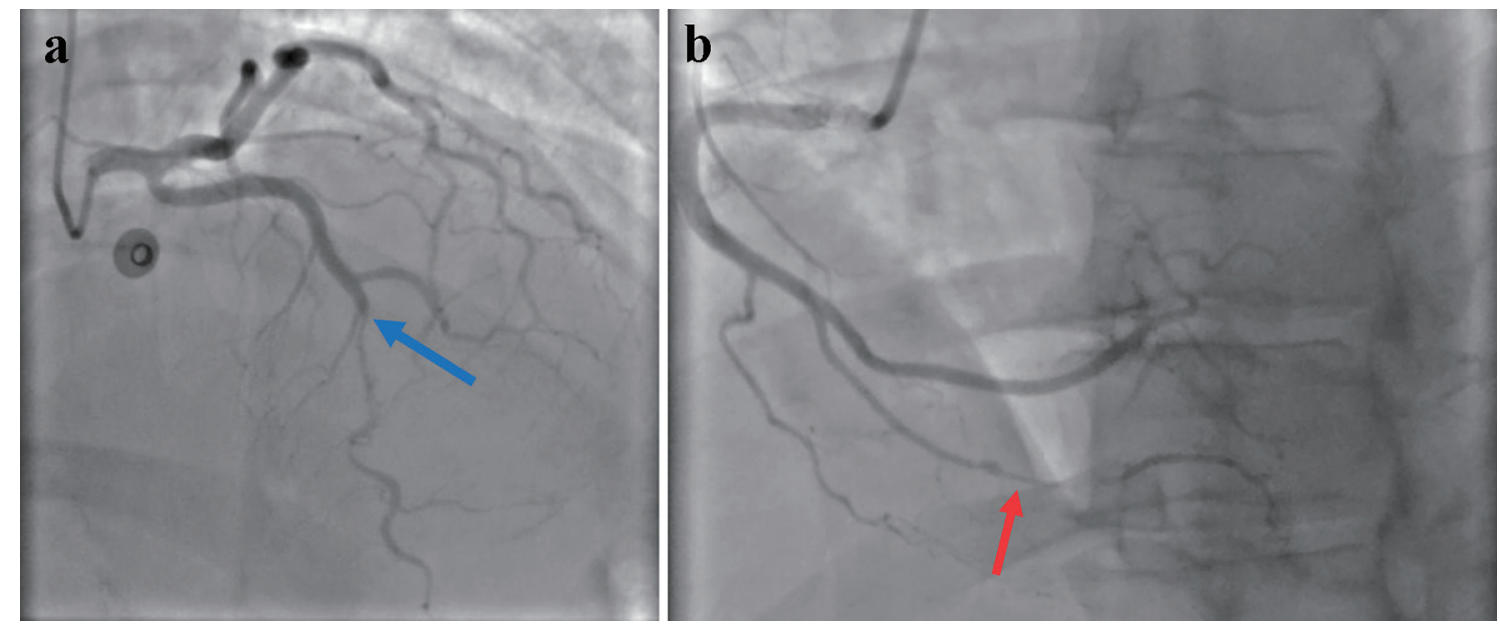

Figure 1. Transradial engagement of the coronary arteries, representing the left and right coronary circuits, respectively. (a) is significant for an otherwise patent left main coronary artery. The mid-portion of the left anterior descending artery, as illustrated by the blue arrow, highlights the segment that is concerning for coronary artery dissection. Similarly, (b) represents the right coronary circuit with a red arrow highlighting the mid/distal PDA with signs suggestive of SCAD. SCAD: spontaneous coronary artery dissection; PDA: posterior descending artery.

stable with a temperature of $36.3{ }^{\circ} \mathrm{C}$, heart rate of 86 beats per minute, oxygen saturation of $96 \%$ on room air, blood pressure of $149 / 73 \mathrm{~mm} \mathrm{Hg}$, and respiratory rate of 18 breaths per minute. The lungs were clear to auscultation bilaterally. Auscultation of the heart was significant for a regular and rhythm without murmurs, rubs, or gallops. The extremities were warm, dry, and with no signs of swelling or pitting edema. The pulses were $2+$ in the radial and dorsalis pedis pulses bilaterally.

\section{Treatment}

Laboratory data on admission were significant for a complete metabolic panel and complete blood count that were within normal limits aside from a potassium of $3.4 \mathrm{mmol} / \mathrm{L}$ (reference 3.5 - $5.0 \mathrm{mmol} / \mathrm{L}$ ). Troponins on admission were $0.45 \mathrm{ng} /$ $\mathrm{mL}$ and progressively peaked at $4.96 \mathrm{ng} / \mathrm{mL}$ (reference range $<0.04 \mathrm{ng} / \mathrm{mL}$ ) $2 \mathrm{~h}$ later. A 12-lead electrocardiogram was significant for sinus rhythm with frequency ectopic beats but otherwise no signs of acute ischemia. Transthoracic echocardiogram was significant for an ejection fraction of $26-30 \%$ without significant valvular disease.

Left heart catheterization was significant for a right dominant circulation with two vessels that demonstrated clear stenosis. The LAD artery was found to be a large caliber vessel with severe diffuse stenosis in the mid vessel that improved distally. The remainder of the LAD, as demonstrated in Figure 1a, appeared narrow and irregular, thus raising the suspicion for SCAD. Similarly, the right coronary artery, as demonstrated in Figure 1b, gave rise to a small caliber posterior descending artery with severe stenosis and TIMI III flow distally. The coronary arteries were not engaged given the suspicious morphology for SCAD. The patient was treated medically with an angiotensin converting enzyme inhibitor (ACEi) and beta blockade with a goal heart rate of less than 60 .

Given the concern for SCAD, the patient underwent fur- ther workup to rule out underlying comorbidities. A magnetic resonance angiogram (MRA) of the head and neck was negative for any signs of aneurysms. MRA of the abdomen was negative for renal artery involvement.

\section{Follow-up and outcomes}

The patient's hospital course remained otherwise unremarkable. The patient remained hemodynamically stable and symptom-free. A repeat transthoracic echocardiogram was significant for an ejection fraction that drastically improved to $60-65 \%$. She was discharged safely to home and advised to continue taking aspirin $81 \mathrm{mg}$, lisinopril $10 \mathrm{mg}$ daily, and metoprolol $50 \mathrm{mg}$ twice daily.

\section{Discussion}

The clinical presentation of SCAD can vary greatly both in symptoms and laboratory workup. Almost invariably, most patients present with symptoms of ACS with correlating cardiac enzymes [5]. Saw et al presented a report of 168 patients with confirmed SCAD who were found to have an average troponin of $6 \mu \mathrm{g} / \mathrm{L}$ (normal $<0.05 \mu \mathrm{g} / \mathrm{L}$ ) [6]. The exact ratio of patients presenting with ST segment elevations or depressions varies greatly but can occur in up to $87 \%$ and $69 \%$ of patients, respectively [7]. Chest pain is the most common symptom and ventricular arrhythmias can be seen in up to $11 \%$ of patients [8].

There are two proposed mechanisms for SCAD - the "outside-in" and "inside-out" hypotheses. The outside-in mechanism, considered to be the most common form, suggests that the intramural hematoma forms within the vessel due to rupture of the vasa vasorum. The inside-out mechanism proposes the reverse in that the tear occurs first leading to a false lumen between the media and tunica intima [9]. 
The gold standard for diagnosing SCAD is coronary angiography. There are four major types of SCAD based on coronary imaging. Type 1 is defined by the connection of a true and false lumen. Type 2, which is also the most common type, is diagnosed when there is no clear connection between the true and false lumens in a coronary spanning at least $20 \mathrm{~mm}$. Type 3 is very similar to type 2 , except the length of the dissection is less than $20 \mathrm{~mm}$. Type $4 \mathrm{SCAD}$ is unique in the sense that there is an obstruction to flow at the distal point of the dissection and hence imitates a thrombus [4].

Despite our advances in medical therapy and clinical suspicion in diagnosis, the true epidemiology remains uncertain as it is still underdiagnosed. Based on the case reports present in the literature, however, we do understand that it is most common in young, peripartum women [5]. The demographic in which it occurs makes diagnosis especially challenging as this patient population is often younger and with few risk factors that would make ACS the likely diagnosis. SCAD occurs more often in females, and can be the cause of acute myocardial infarction in as many as $30 \%$ of cases in women under the age of 50 [10]. Furthermore, it is the most common cause of myocardial infarction (MI) in pregnancy associated with MI [11]. SCAD has been found to be more common in patients with fibromuscular dysplasia as well as other arteriopathies [12]. Despite the predilection, the pathophysiologic association of SCAD with pregnancy is not well understood. As will be discussed, uncontrolled hypertension is the most common cause for recurrence of SCAD but has not been clearly identified as the primary cause.

The coronary predisposition of SCAD is well understood. It most commonly occurs in the LAD artery. The diagonal and septal branches are affected in 46-61\% of cases, and the circumflex, ramus, and marginal branches are affected in $15-45 \%$ of cases. When the right coronary artery is affected, it most commonly occurs in acute marginal, posterior descending, and posterolateral branches in up to $39 \%$ of cases [4]. SCAD affecting multiple coronary vessels is rare but can occur in $9-23 \%$ of cases, depending on the literature [4].

As with any kind of vascular insufficiency, the primary goal in the acute setting is to preserve myocardial perfusion. But in the case of SCAD, sometimes less is more. Depending on the severity and extent of SCAD, most cases can be managed conservatively without percutaneous coronary intervention (PCI). Hassan et al have demonstrated in a review that greater than $95 \%$ of patients were found to have signs of angiographic recovery 30 days post diagnosis with medical therapy alone [13]. Cardiac catheterization poses inherent risks with wire manipulation and balloon deployment that risk further damaging the already compromised coronary artery. Likewise, thrombolytic therapy is also questionable as it can potentially precipitate bleeding in the pericardial sac or worsen the intramural hematoma [14].

At this time, there is a lack of clinical trials to establish the ideal guideline-directed medical therapy of SCAD. However, the basic principles currently employed focus on promoting flow distal to the lesion and minimizing exacerbation of the coronary dissection. Hypertension is a clear risk factor and known to cause recurrent SCAD [6]. The preferred agents depend on the underlying comorbidities. If the patient has dias- tolic heart failure, then angiotensin converting enzyme inhibitors or angiotensin receptor blockers should be used [6]. Beta blockade is also a promising agent as it can also decrease contractility [5]. Lipid lowering therapy, with statins or fibrates, is often reserved for patients with underlying dyslipidemias as there is no clear benefit in isolated SCAD [15]. Systemic anticoagulation and dual antiplatelet therapy both have limited benefit as they can potentially exacerbate bleeding into the intramural hematoma. Thus, their use is reserved for specific cases with persistent thrombus burden [16].

Patients that have been diagnosed with SCAD have a high risk of recurrence. Based on the setting and underlying comorbidities, the reported rates in the literature range from $10 \%$ to $30 \%$ [4]. Saw et al published a report in which 327 patients with SCAD were followed prospectively. Nearly $83 \%$ of patients were managed medically and $10.4 \%$ of all patients were found to have recurrent SCAD. As previously mentioned, hypertension increased the risk of recurrence (hazard ratio 2.46, $\mathrm{P}=0.011$ ) [6]. Unfortunately, the average age of patients was 52 and, given that SCAD often affects young females, more reports are needed to better understand the long-term outcomes of different patient populations.

\section{Learning points}

SCAD is becoming a more commonly diagnosed cause of acute myocardial infarction, especially in young female patients with few cardiovascular risk factors. Although a clear correlation exists between SCAD and female gender, pregnancy, and underlying arteriopathies, the clear pathophysiological mechanism remains to be determined. SCAD is best managed with a conservative approach to minimize the risk of mitigating vascular damage. SCAD can occur in a patient of any demographic and has several unique findings upon presentation. The medical community will benefit from further research to establish guideline-directed medical therapy on management of this increasingly prevalent condition.

\section{Acknowledgments}

None to declare.

\section{Financial Disclosure}

None to declare.

\section{Conflict of Interest}

None to declare.

\section{Informed Consent}

Informed consent was obtained. 


\section{Author Contributions}

Anton Mararenko: preparation of manuscript. Greg Minassian: preparation of manuscript. Anshu Kataria: preparation of manuscript. Firas Ajam: preparation of manuscript. Matthew Schoenfeld: senior author, operating attending.

\section{Data Availability}

The data supporting the findings of this study are available from the corresponding author upon reasonable request.

\section{References}

1. Kim ESH. Spontaneous coronary-artery dissection. N Engl J Med. 2020;383(24):2358-2370.

2. Boulmpou A, Kassimis G, Zioutas D, Meletidou M, Mouselimis D, Tsarouchas A, Tzikas S, et al. Spontaneous Coronary Artery Dissection (SCAD): case series and mini review. Cardiovasc Revasc Med. 2020;21(11):14501456.

3. Alquran L, Patel A, Safi L, Patel A. A rare case of multivessel SCAD successfully treated with conservative medical management. Case Rep Cardiol. 2020;2020:8468730.

4. Hayes SN, Tweet MS, Adlam D, Kim ESH, Gulati R, Price JE, Rose CH. Spontaneous coronary artery dissection: JACC State-of-the-Art review. J Am Coll Cardiol. 2020;76(8):961-984.

5. Tweet MS, Hayes SN, Pitta SR, Simari RD, Lerman A, Lennon RJ, Gersh BJ, et al. Clinical features, management, and prognosis of spontaneous coronary artery dissection. Circulation. 2012;126(5):579-588.

6. Saw J, Humphries K, Aymong E, Sedlak T, Prakash R, Starovoytov A, Mancini GBJ. Spontaneous coronary artery dissection: clinical outcomes and risk of recurrence. J Am Coll Cardiol. 2017;70(9):1148-1158.

7. Lettieri C, Zavalloni D, Rossini R, Morici N, Ettori F, Leonzi O, Latib A, et al. Management and long-term prognosis of spontaneous coronary artery dissection. Am J Cardiol. 2015;116(1):66-73.
8. Lindor RA, Tweet MS, Goyal KA, Lohse CM, Gulati R, Hayes SN, Sadosty AT. Emergency department presentation of patients with spontaneous coronary artery dissection. J Emerg Med. 2017;52(3):286-291.

9. Ravipati H, Rodrigues S, Rao S, Hatharaliyadda B, Junia $C$. The young heart tears easily apart: a case report of spontaneous coronary artery dissection. Cureus. 2021;13(6):e15590.

10. Meng PN, Xu C, You W, Wu ZM, Xie DJ, Zhang H, Pan C, et al. Spontaneous coronary artery dissection as a cause of acute myocardial infarction in young female population: a single-center study. Chin Med J (Engl). 2017;130(13):1534-1539.

11. Elkayam U, Jalnapurkar S, Barakkat MN, Khatri N, Kealey AJ, Mehra A, Roth A. Pregnancy-associated acute myocardial infarction: a review of contemporary experience in 150 cases between 2006 and 2011. Circulation. 2014;129(16):1695-1702.

12. Olin JW, Froehlich J, Gu X, Bacharach JM, Eagle K, Gray BH, Jaff MR, et al. The United States registry for fibromuscular dysplasia: results in the first 447 patients. Circulation. 2012;125(25):3182-3190.

13. Hassan S, Prakash R, Starovoytov A, Saw J. Natural history of spontaneous coronary artery dissection with spontaneous angiographic healing. JACC Cardiovasc Interv. 2019;12(6):518-527.

14. Shamloo BK, Chintala RS, Nasur A, Ghazvini M, Shariat P, Diggs JA, Singh SN. Spontaneous coronary artery dissection: aggressive vs. conservative therapy. J Invasive Cardiol. 2010;22(5):222-228.

15. Arnett DK, Blumenthal RS, Albert MA, et al. 2019 ACC/AHA guideline on the primary prevention of cardiovascular disease: executive summary: a report of the American College of Cardiology/American Heart Association Task Force on clinical practice guidelines [published correction appears in J Am Coll Cardiol. 2019 Sep 10;74(10):1428-1429] [published correction appears in J Am Coll Cardiol. 2020 Feb 25;75(7):840]. J Am Coll Cardiol. 2019;74(10):1376-1414.

16. Shah R, Khan B, Latham SB, Khan SA, Rao SV. A metaanalysis of aspirin for the primary prevention of cardiovascular diseases in the context of contemporary preventive strategies. Am J Med. 2019;132(11):1295-1304 e1293. 